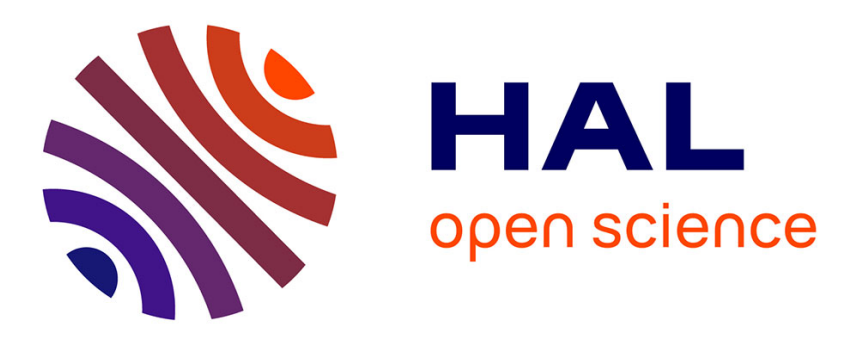

\title{
Role of the resonator geometry on the pressure spectrum of reed conical instruments
}

Jean Kergomard, Philippe Guillemain, Patrick Sanchez, Christophe Vergez, Jean-Pierre Dalmont, Bruno Gazengel, Sami Karkar

\section{To cite this version:}

Jean Kergomard, Philippe Guillemain, Patrick Sanchez, Christophe Vergez, Jean-Pierre Dalmont, et al.. Role of the resonator geometry on the pressure spectrum of reed conical instruments. 2019. hal-01865994v2

\author{
HAL Id: hal-01865994 \\ https://hal.science/hal-01865994v2
}

Preprint submitted on 1 Feb 2019

HAL is a multi-disciplinary open access archive for the deposit and dissemination of scientific research documents, whether they are published or not. The documents may come from teaching and research institutions in France or abroad, or from public or private research centers.
L'archive ouverte pluridisciplinaire HAL, est destinée au dépôt et à la diffusion de documents scientifiques de niveau recherche, publiés ou non, émanant des établissements d'enseignement et de recherche français ou étrangers, des laboratoires publics ou privés. 


\title{
Role of the resonator geometry on the pressure spectrum of reed conical instruments
}

\author{
Jean Kergomard, Philippe Guillemain, Patrick Sanchez, Christophe Vergez ${ }^{1}$, \\ Jean-Pierre Dalmont, Bruno Gazengel ${ }^{2}$, Sami Karkar ${ }^{3}$, \\ ${ }^{1}$ Aix Marseille Univ, CNRS, Centrale Marseille, LMA, \\ UMR 7031, Marseille, France \\ ${ }^{2}$ LUNAM Université, Université du Maine, Unité Mixte de Recherche CNRS 6613, \\ Avenue Olivier Messiaen, 72085 Le Mans Cedex 9, France \\ ${ }^{3}$ Univ Lyon, Ecole Centrale de Lyon, ENISE, ENTPE, CNRS, \\ Laboratoire de Tribologie et Dynamique des Systèmes LTDS, \\ UMR5513, F-69134, ECULLY, France
}

\section{Summary}

\begin{abstract}
Spectra of musical instruments exhibit formants or anti-formants which are important characteristics of the sounds produced. In the present paper, it is shown that anti-formants exist in the spectrum of the mouthpiece pressure of saxophones. Their frequencies are not far but slightly higher than the natural frequencies of the truncated part of the cone. To determine these frequencies, a first step is the numerical determination of the playing frequency by using a simple oscillation model. An analytical analysis exhibits the role of the inharmonicity due to the cone truncation and the mouthpiece. A second step is the study of the input impedance values at the harmonics of the playing frequency. As a result, the consideration of the playing frequency for each note explains why the anti-formants are wider than those resulting from a Helmholtz motion observed for a bowed string. Finally numerical results for the mouthpiece spectrum are compared to experiments for three saxophones (soprano, alto and baritone). It is shown that when scaled by the length of the missing cone, the antiformant frequencies in the mouthpiece are very similar for the three instruments. The frequencies given by the model are close to the natural frequencies of the missing cone length, but slightly higher. Finally, the numerical computation shows that anti-formants and formants might be found in the radiated pressure.
\end{abstract}

\section{1 Introduction}

The auditory recognition of musical instruments is a rather intricate issue. It is generally admitted that the existence of formants is an important element that contributes to the identification of an instrument. A formant (resp. an anti-formant) can be defined as a frequency band reinforced (resp. attenuated) whatever the played note. Formants are in general regarded as an important characteristic of the tone colour (or of the vowels in speech). It needs to be distinguished from other timbre characteristics, such as the weakness of harmonics of a given rank (e.g., the even harmonics in the clarinet sound). The statement of the problem is ancient $[4,5]$. Smith and Mercer [4] found formants produced by conical instruments similar to saxophones. Benade [2] wrote: "There is in fact almost no simple formant behavior to be recognized in the sound production of wind instruments". However several authors observed that the spectrum of the acoustic pressure in the reed of a bassoon [1] or in the mouthpiece of a saxophone $[2,3]$ is close to the function $\sin (\mathrm{nq}) / \mathrm{nq}$, where $n$ is the harmonic number and $q$ can be determined experimentally.

This implies that anti-formants can appear around frequencies satisfying $\sin (n q)=0$. If formants (or anti-formants) exist, a consequence of the above mentioned definition is that their frequencies cannot depend on the length of the tube for a given note. Conversely they depend either on other geometrical parameters (length of the missing cone, input radius, apex angle of the truncated cone, dimensions of the mouthpiece, geometry of the toneholes) or on the excitation parameters.

The simplest model, based upon the analogy with bowed string instruments, was studied by many authors $[6,7,8,9,10,11,12]$, and a result is the waveshape approximation of the mouthpiece pressure by a rectangle signal, i.e., the waveshape of the ideal Helmholtz motion. Formerly, some authors explained that an approximation of the natural frequency of reed conical instruments is equal to that of an "openopen" cylinder whose length is the length of the truncated cone extended to its apex $[13,14,15]$. Because 


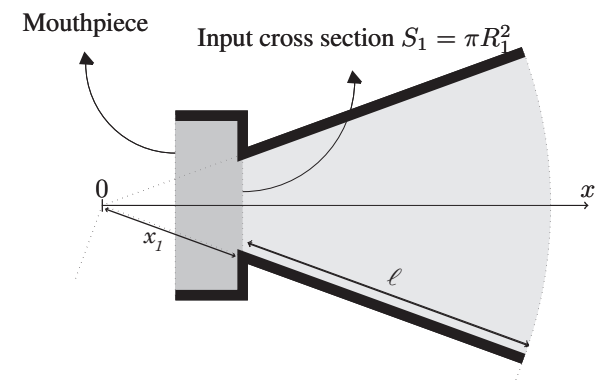

Figure 1: Notations for the geometrical parameters. For a soprano saxophone, the length of the missing part of the cone is approximately $x_{1}=0.126 \mathrm{~m}$. Typical values of the coefficient $\beta$ are included in the interval $[0.13,0.3]$.

the length of the missing cone does not vary with the note, a consequence of the analogy is that the duration of the negative pressure episode is common to all notes. Another consequence is the existence of anti-formants close to the natural frequencies of the missing part of the truncated cone (which is denoted $x_{1}$ in the present paper, see Fig. 1 for the notations).

The analogy with the Helmholtz motion of bowed strings leads to the result that in the function $\sin (n q) / n q, q \simeq \pi \beta$, where $\beta$ is the ratio of the short length of the string to its total length. For a truncated cone, $\beta$ is the ratio of the length of the missing cone $x_{1}$ to the total length $x_{2}=\ell+x_{1}$ :

$$
\beta=\frac{x_{1}}{x_{1}+\ell}=\frac{x_{1}}{x_{2}}=\frac{R_{1}}{R_{2}}
$$

$R_{1}$ and $R_{2}$ are the radii at abscissae $x_{1}$ and $x_{2}$, respectively.

It is still the only model that yields analytical expressions for the sound produced, and therefore it is used as a reference for the present study. In a paper written by some of the present authors, it was shown that a simple numerical model can largely improve the model of the Helmholtz motion [16]. We call it the "Reed-Truncated-Cone" model (RTC model). The difference between the two models lies in the resonator model. Example of waveshapes obtained with the two models are shown in Fig. 2. Using the RTC model for the present investigation on the spectrum, the paper aims at further understanding of the existence of formants or anti-formants in the mouthpiece pressure spectrum, and, to some extent, of the external pressure. The computation is done ab initio in the time domain.

The study is limited to the first register, which resembles the Helmholtz motion (periodic regime, one positive pressure and one negative pressure episodes). The RTC model is based upon the observation that in practice the mouthpiece volume is approximately equal to that of the missing cone [17], entailing a weak

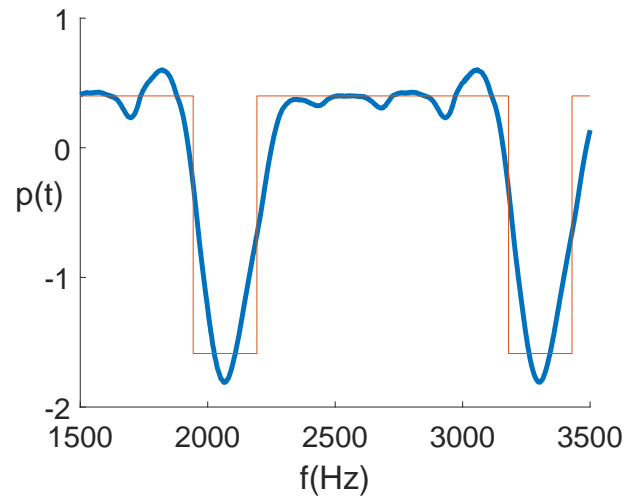

Figure 2: Example of waveshape for a soprano saxophone $\left(x_{1}=0.126 \mathrm{~m}\right.$ and $\left.\ell=0.55 \mathrm{~m}\right)$ for the excitation parameters $\gamma=0.4, \zeta=0.65$ (see Sect. 3.1). Thick line: RTC model; thin line: ideal Helmholtz motion model.

inharmonicity for the lower notes. The resonator is a truncated cone, of length $\ell$, with a pure lumped compliance at its input (that of the air in the mouthpiece volume). This is a simplification, because in some instruments, such as the oboe, the cone of the resonator can be more complicated, with two different tapers, entailing a further reduction of inharmonicity [18]. The double taper is not considered here, because the waveform of the internal pressure given by the RTC model seems to compare well enough with experimental waveforms [16].

The effects of wall losses and radiation are ignored. The model of toneholes is extremely simplified: for a given fingering with a given number of toneholes, the resonator is assumed to be equivalent to a truncated cone of equivalent length $\ell$. Therefore, for a given note, two parameters are sufficient, the length $\ell$ and the radius ratio $R_{2} / R_{1}$ (actually, without losses, it is not necessary to define the values of the two radii, or the apex angle). Therefore, according to the hypotheses adopted in the RTC model, the length of the missing cone is expected to be predominant in the dependence of the frequencies of formants and antiformants.

As an intermediate step, the paper attempts to determine more precise values for the first playing frequency, because it has an influence on the spectrum, as discussed later. This influence entails the dependence of the pressure spectra on the fingering, i.e., on the length $\ell$, and the enlargement of the formants.

In Sect. 2 the RTC model is presented for the resonator, with the calculation of the transfer functions of the resonator (between input and output quantities). Sect. 3 recalls some known results about the "cylindrical saxophone" model, which is similar to that of an ideal bowed string, and gives the classical solution of the Helmholtz motion. The paradox of the analogy between a conical instrument and a 
cylindrical saxophone is discussed.

Then, in Sec. 4, it is shown how the playing frequencies for a truncated cone with mouthpiece differ from those corresponding to the ideal Helmholtz motion, because they depend on the excitation parameters, and on the note.

In Sec. 5 the zeros of the transfer functions are investigated with their dependence on the playing frequencies.

In Section 6, thanks to the results of numerical computations obtained with the RTC model [16] of the sound production, the frequencies of the minima of the sampled input impedance are compared to those of the mouthpiece pressure, and the existence of formants and anti-formants is discussed in both the internal pressure and the external one.

In Section 7 experimental results are presented, and compared to the numerical results.

\section{Basic model of the resonator}

\subsection{Resonator model of the RTC.}

A truncated cone is considered (see Fig. 1), provided with a mouthpiece of volume equal to the volume of the missing cone: $V=x_{1} S_{1} / 3$. The mouthpiece is assumed to be small with respect to the wavelength. The shunt acoustic compliance of the mouthpiece is $V / \varrho c^{2}$. The inertia of the air within the mouthpiece (i.e. the series acoustic mass), is ignored, because the sound production by reed instruments occurs at frequencies close to impedance maxima (this is discussed in Ref. [16]). At abscissae $x_{1}$ and $x_{2}$, the cross-section areas are $S_{1}$ and $S_{2}$, respectively. No resonator losses are considered, and the output impedance of the cone is assumed to be zero. This implies that the radiation reactance is zero too: it could be taken into account by a slight modification of the length of the truncated cone. In the frequency domain, the solution of the acoustic equations in the conical tube can be written as the sum of two spherical, travelling pressure waves $P^{ \pm}(x)$ (see e.g. [19]):

$$
\begin{array}{r}
P(x)=P^{+}(x)+P^{-}(x) ; \\
U(x)=\frac{S(x)}{\rho c}\left(P^{+}(x)-P^{-}(x)+\frac{P(x)}{j k x}\right) \\
P^{ \pm}=a^{ \pm} \exp (\mp j k x) / x .
\end{array}
$$

$P(x)$ is the pressure and $U(x)$ is the flow rate. $k=$ $2 \pi f / c$ is the wavenumber, $f$ is the frequency, $c$ the speed of sound, $\rho$ the air density. Standard transfer matrices for the lumped compliance and the truncated cone are used for this model in the frequency domain. Because the pressure $P_{2}$ at the output is zero, the two following transfer functions between the mouthpiece input quantities (pressure $P$ and flow rate $U$ ) and the output flow rate $U_{2}$ are found:

$$
\begin{gathered}
P=\frac{j \varrho c}{\pi R_{1} R_{2}} \sin (k \ell) U_{2}, \\
U=\frac{R_{1}}{R_{2}}\left\{\cos (k \ell)+\sin (k \ell) /\left(k x_{1}\right)\right. \\
\left.-\sin (k \ell) k x_{1} / 3\right\} U_{2}
\end{gathered}
$$

These transfer functions have zeros, but no poles. At the frequencies of the zeros, because $U_{2}$ is finite, the input quantities $P$ and $U$ vanish. The external pressure can be derived from the output flow rate $U_{2}$, which at low frequencies can be regarded as a monopole source. Omitting the delay, the low frequency relationship between the external pressure at distance $d$ and the output flow rate is the following:

$$
P_{e x t}=j \omega \rho U_{2} \frac{1}{4 \pi d} .
$$

$\omega$ is the angular frequency. For our purpose, we have interest in the physical quantities $P, U$ and $U_{2}$, which depend on the excitation, as well as the extrema of the two transfer functions, which depend on the resonator only. The zeros of the transfer functions for the pressure and flow rate (Eqs. (5) and (6)) are the zeros and poles, respectively, of the input impedance:

$$
Z=\frac{\rho c}{S_{1}} \frac{j \sin (k \ell)}{\cos (k \ell)+\sin (k \ell) /\left(k x_{1}\right)-\sin (k \ell) k x_{1} / 3} .
$$

\subsection{Comparison with the "cylindrical saxophone" model}

A further approximation of the RTC model is the classical cylindrical saxophone model. The function $1 / x-x / 3$ is identified with the expansion of the function $\cot (x)$. The transfer function equation (6) is unchanged, and, under the following condition,

$$
k x_{1}=2 \pi x_{1} / \lambda<<1,
$$

where $\lambda$ is the wavelength, Eq. (6) becomes:

$$
U=\frac{R_{1}}{R_{2}}\left[\cos (k \ell)+\sin (k \ell) \cot \left(k x_{1}\right)\right] U_{2} .
$$

The input impedance becomes:

$$
Z=\frac{j \varrho c}{S_{1}} \frac{\sin (k \ell) \sin \left(k x_{1}\right)}{\sin \left[k\left(\ell+x_{1}\right)\right]} .
$$

This formula is equivalent to that of the admittance of a string at the bow position. Therefore the Helmholtz motion is a particular solution of the self-sustained oscillation problem. We call this model "cylindrical saxophone" model (however for a cylinder $R_{1}=R_{2}$, while here the radii $R_{1}$ and $R_{2}$ are different). Comparing Eqs. (6) and (10), it can be noticed that in the transformation, an infinity of poles have been added, entailing different behaviours of the transfer functions and input impedance. 


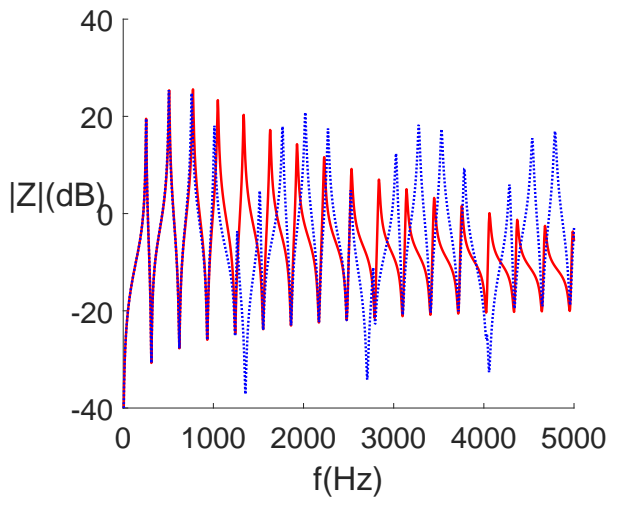

Figure 3: Example of input impedance modulus curves (dB defined by $20 \log \left(\left|Z S_{1} / \rho c\right|\right)$ for $x_{1}=$ $0.126 \mathrm{~m}$ and $\ell=0.55 \mathrm{~m}$. Solid line (red online): RTC model; dotted line (blue online): approximation corresponding to the cylindrical saxophone model (ideal Helmholtz motion).

Formula (11) exhibits that there are two kinds of input impedance dips: i) the solutions of $\sin (k \ell)=$ 0 , which depend on the note; ii) the solutions of $\sin \left(k x_{1}\right)=0$, which do not depend on the note. Fig. 3 shows an example of input impedance curve. For this figure, realistic visco-thermal losses (for an average cone radius) have been taken into account in Eq. (11). The two kinds of dips appear. We add three remarks:

1. The case shown in Fig. 3 corresponds to an irrational value of the parameter $\beta$. For rational values of $\beta$, the frequencies of the second kind of dips for the cylindrical saxophone can coincide with those of the truncated cone, but losses make the dips distinct.

2. The resonances of the cylindrical saxophone are perfectly harmonic (see the dotted lines in Fig. 3). The figure exhibits that this is not the case for the truncated cone with mouthpiece (solid line in Fig. 3). For the RTC model the second kind of minima disappears, according to Eq. (8). The comparison between the RTC model and the cylindrical saxophone model shows the effect of inharmonicity. It will be shown in Sections 4 and 5 that, as a consequence, minima close to dips of the cylindrical saxophone appear in the input impedance at the harmonics of the playing frequency. For these harmonics, we call the input impedance curve the sampled impedance (see [20]).

3. These minima are responsible for anti-formants of the input pressure, because their frequencies depend few of the note.

\section{Oscillation model and the so- lution of the ideal Helmholtz motion}

\subsection{Helmholtz motion}

The complete oscillation model is now investigated for the cylindrical saxophone. For the exciter (mouth and reed), the model used was presented in Ref. [16]. The nonlinear characteristic is deduced from the model established by Wilson and Beavers [21]. Nevertheless no reed dynamics is considered. Two dimensionless parameters were defined by these authors: the mouth pressure $\gamma$ and the reed opening $\zeta$ at rest (in Ref. [21], the parameters are the same, with different notations). The model is based upon the stationary Bernoulli law and some hypotheses, with a localized non-linearity. With the approximation (11) for the impedance, analytical solutions exist for the oscillations, in particular the so-called Helmholtz motion [9], which is a rectangle signal.

Using the subscript $H$ for the Helmholtz motion, the fundamental frequency is $f_{H 1}=c / 2\left(l+x_{1}\right)$ (the wavelength is twice the total length of the cone). The frequency $f_{H n}$ of the $n$th harmonic is given by:

$$
f_{H n}=\frac{n c}{2\left(\ell+x_{1}\right)} .
$$

The value of the signal during the longer episode is $\gamma$ (when the reed does not close the mouthpiece), while the value during the shorter episode is $-(1-$ $\beta$ ) $\gamma / \beta$ (when the reed closes the mouthpiece, for the definition of $\beta$, see Eq. (1)). This case corresponds to the condition $\gamma>\beta$, which is often satisfied in practice at least for the lowest notes (see Ref. [9]), as well for the choice of parameters in the theoretical part of the present paper. The spectrum components of the input pressure $p(t)$ are as follows:

$$
\begin{aligned}
P_{n} & =-\gamma(-1)^{n} \frac{\sin X_{n}}{X_{n}} \\
X_{n} & =2 \pi \frac{f_{H n} x_{1}}{c}=k_{H n} x_{1} \\
& =\frac{n \pi x_{1}}{\ell+x_{1}}=n \pi \beta .
\end{aligned}
$$

Here, and in what follows, the pressure is dimensionless: all pressures in the resonator are divided by the reed closure pressure $p_{M}$, which is proportional to the reed stiffness. The waveshape and the relative pressure spectrum are independent of the excitation parameters. The flow rate $u(t)$ at the input is constant, in order for the input average power per period to vanish. For frequencies $f_{m}=m c /\left(2 x_{1}\right)$, $\sin X_{m}=\sin (m \pi)=0$ : there is a zero in the pressure spectrum, under the condition that $m / n=\beta$ is rational. If $\beta$ is irrational, there is a minimum amplitude near the frequencies $f_{m}$. As a consequence, whatever the cone length $\ell$, there is an amplitude minimum around these frequencies, i.e., an anti-formant, and 
these frequencies are the natural frequencies of the length $x_{1}$ of the missing cone.

Writing $x_{1}=\left(\ell+x_{1}\right)-\ell$, Eq. (13) implies:

$$
\begin{array}{r}
\sin X_{n}=(-1)^{n} \sin \left(n \pi \ell /\left(\ell+x_{1}\right)\right) \\
=(-1)^{n} \sin \left(k_{H n} \ell\right),
\end{array}
$$

thus Eqs. (6) and (13) give the amplitude of the output flow rate:

$$
U_{2, n}=\frac{\gamma}{X_{n}} \frac{\pi R_{1} R_{2}}{\varrho c}
$$

There are no zeros in the spectrum of the output flow rate. Eqs. $(7,18)$ show that the spectrum of the external pressure $P_{\text {ext }}$ is constant and the signal is a Dirac comb. Neither formants nor anti-formants exist in the radiated pressure $P_{\text {ext }}$.

\subsection{Comparison of a cylindrical saxo- phone with a truncated cone}

The present study was motivated by a paradox presented in a conference paper by some authors of the present article [23], and summarized hereafter.

For bassoon sounds, Gokhstein [7] showed both experimentally and theoretically that the duration of reed closure is independent of the played note, i.e., of the equivalent length of the resonator. This duration is related to the round trip of a wave over a length equal to that of the missing part of the cone $x_{1}$. The corresponding frequency is the natural frequency of this length $c /\left(2 x_{1}\right)$. This seems to validate the analogy with the bowed string excited at a given length of the bridge (or with the cylindrical saxophone, which is also analogous to a kind of stepped cone [10]). This was studied in several papers $[8,9,10]$. However the analogy is known to be valid only if the length of the missing cone is small compared with the wavelength (see Condition (9)). This condition is not fulfilled for the natural frequency of the missing part, which is equal to the half of the corresponding wavelength.

Thanks to the bowed string analogy, useful conclusions can be drawn concerning important features of the sound production, such as oscillation regimes and amplitudes. A priori accurate insight of the tone color for higher frequencies, which do not fullfil the condition (9), cannot be expected. Nevertheless measured spectra of the internal pressure of saxophones exhibit minima [22] at frequencies corresponding roughly to the harmonics of the fundamental frequency $c /\left(2 x_{1}\right)$. On the one hand this is an argument in favour of the analogy with the Helmholtz motion, while on the other hand this result is paradoxical because for these frequencies, the condition (9) is not fulfilled. It will be shown how inharmonicity of the resonator, which exists neither in a perfect string nor in a cylindrical saxophone, plays a major role in a real conical instrument. In particular it implies that the playing fre- quency differs from natural frequencies $c /\left(2\left(x_{1}+\ell\right)\right)$ of the complete cone.

In order to make easier the comparison of the results for a truncated cone with those for the Helmholtz motion, we define a quantity proportional to the external pressure (Eq. (7)) and inversely proportional to the blowing pressure, i.e., to the square root of the radiated power, as follows:

$$
W=U_{2} \frac{\varrho c}{\pi R_{1} R_{2} \gamma} k x_{1} .
$$

We call $W$ the normalized output flow rate. For the Helmholtz motion and the harmonics of the playing frequency, which is our reference, $|W|$ is unity (see Eqs. (18) and (15)). For the truncated cone, we redefine the transfer functions ( 5 and 6$)$, as follows:

$$
\left(\begin{array}{c}
P \\
U
\end{array}\right)=\left(\begin{array}{l}
F_{p} \\
F_{u}
\end{array}\right) W
$$

with

$$
\begin{gathered}
F_{p}=\frac{j \gamma}{k x_{1}} \sin (k \ell), \\
F_{u}=\frac{S_{1}}{\rho c} \frac{\gamma}{k x_{1}}\left\{\left(\cos (k \ell)+\sin (k \ell) /\left(k x_{1}\right)\right.\right. \\
\left.-\sin (k \ell) k x_{1} / 3\right\} .
\end{gathered}
$$

\section{Playing frequency of a conical instrument}

The playing frequency is a compromise between the different modes of the resonator and varies with the excitation parameters (see especially [24, 25]). For a truncated cone, the playing frequencies slightly differ from the resonance frequencies of the cylindrical saxophone, and the consequences for the pressure spectrum are significant. In the present section the values of the playing frequency are studied. Then, in section 5 the dependence of the formants and anti-formants on the playing frequency is investigated.

It is often considered that the playing frequencies are very close to the natural frequencies of the resonator. However several causes of discrepancies between playing and natural frequencies were recently investigated for reed cylindrical instruments [26]. Among them there is the effect of inharmonicity of the resonator for conical instruments, which are truncated cones. The effect of the truncation is important, even if it is limited by a proper choice of the mouthpiece dimensions. When the approximation of the cylindrical saxophone is abandoned, the playing frequencies differ from the natural frequencies of the total length $\ell+x_{1}$ (Eq. (12)).

\subsection{Numerical estimation of the play- ing frequencies (RTC model)}

Using the numerical RTC model, including the excitation model and the resonator model corresponding 


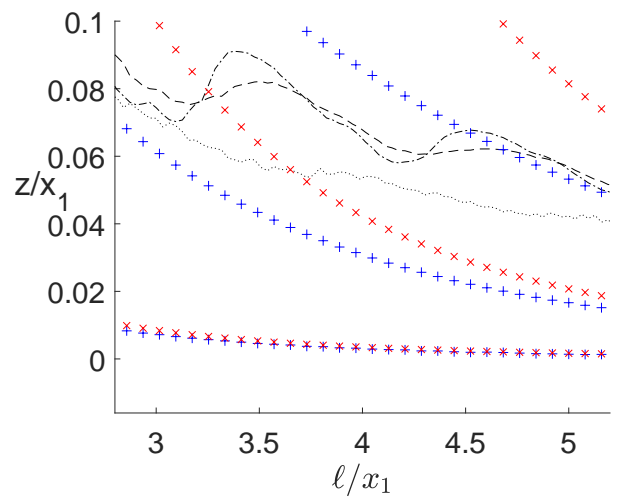

Figure 4: Length $z(-z$ is the length correction) related to the playing frequency represented by the ratio $\ell / x_{1}$ for several values of the length $\ell$ of the truncated cone (simulation results). When $\ell$ varies from $0.35 \mathrm{~m}$ to $0.67 \mathrm{~m}$, the ratio $\beta$ decreases from 0.26 to $0.16 . \quad x_{1}=0.126 \mathrm{~m}$. Thin, black lines: dotted $(\gamma=\zeta=0.4)$, mixed $(\gamma=0.45 ; \zeta=0.85)$, dashed $(\gamma=0.4 ; \zeta=0.65)$. +++ -blue online) Formula $(25)$, for one, two, three terms of the series (from bottom to top). xxx (red online) Formula (26), for one, two, three terms of the series (from bottom to top).

to Eq. (8), the playing frequency of the first periodic regime was determined. In order to calculate the playing frequency, we seek the number of samples between two changes in sign of the input pressure (when the pressure is negative and becomes positive). The typical number of samples for one period is larger than 1000. The relative error on the total equivalent length is less than $0.1 \%$, and that on the length correction is less than $1 \%$.

It is convenient to represent the shift between the playing frequency $f_{p}$ and that of the ideal Helmholtz motion by a length correction, denoted $-z$, as follows

$$
k_{p}=\frac{2 \pi f_{p}}{c}=\frac{\pi}{\ell+x_{1}-z} .
$$

$z=0$ corresponds to the case where these two frequencies are equal. The thin lines in Fig. 4 show, for three pairs of $(\gamma, \zeta)$, that the length correction is negative, entailing that the playing frequency is higher than the first resonance frequency. The length $z$ is significantly smaller than the length $x_{1}$ of the missing cone, and consequently much smaller than the total length, whatever the value of the cone length $\ell$. However the comparison of the classical approximation of the resonance frequency $c / 2 /\left(\ell+x_{1}\right)$ and the playing frequency shows that the difference between them is not negligible: $4 \%$ for $\ell=0.35 \mathrm{~m}$, i.e., 60 cents, and $1 \%$ for the lowest note $(\ell=0.67 \mathrm{~m})$, i.e., 15 cents.

For dimensions close to those of a soprano saxophone, the choice of $0.35 \mathrm{~m}$ as the shortest value for the cone length is due to the difficulty for finding a periodic regime with the ab initio computation and a short $\ell$. The playing frequencies are in the range $[209 \mathrm{~Hz}, 438 \mathrm{~Hz}]$ for $c=340 \mathrm{~ms}^{-1}$. The issue of the regime stability is complicated, and is out of the scope of the present paper (see $[9,12,27]$ ).

\subsection{Analytical estimation of the play- ing frequencies}

In order to understand the role of inharmonicity in the playing frequency, the influence of the second resonance frequency, which is higher than twice the first, and that of the third one, can be estimated in a quantitative way. For this purpose, the result due to Boutillon [28] is used, valid under the condition that the reed dynamics is ignored. With this condition, this is one of the equations of the Harmonic Balance Method (HBM, see for an explanation [19, p. 518]), therefore it does not need the computation of the transient. Considering that the length correction depends little on the excitation parameters, the spectrum of the input pressure is approximated by its value for the Helmholtz motion, and it is possible to find analytically an order of magnitude of the length correction. The "reactive power rule" leads to the equation to be solved for the unknown playing frequency, denoted $\omega$ :

$$
\sum_{n} n\left|P_{n}\right|^{2} \operatorname{Im}[Y(n \omega)]=0 .
$$

$P_{n}$ is given by Eq. (13). In Appendix A, two approximate methods of calculation for the corresponding length correction $-z$ are used. The first one gives the result:

$$
z=\frac{\sum_{n} z_{n} n^{2} \sin ^{2}(n \pi \beta) / \operatorname{Res}_{n}}{\sum_{n} n^{2} \sin ^{2}(n \pi \beta) / R e s_{n}},
$$

where $z_{n}$ is the length correction corresponding to the $n t h$ resonance frequency and $R e s_{n}$ the residue of this resonance in the formula (8) of the input impedance. If the lengths $z_{n}$ were equal for all resonance frequencies (no inharmonicity), the correction for the playing frequency would be equal to them.

Fig. 4 compares the numerical results with those obtained using the two formulas (25) and (26, see hereafter). For the first one, the main features are the correct order of magnitude when more than one term are kept in Eq. (25), and the global decrease when the length $\ell$ increases. The difference between the results with 1 and 2 terms exhibits the importance of the inharmonicity between the first two resonances, due to the truncation of the cone (the result limited to one term is nothing else than the length correction for the first resonance). It appears that the playing frequency obtained from the numerical computation lies between the results of Eq. (25) for 2 and 3 harmonics (i.e., for 2 and 3 terms of the series). The calculation with 4 terms gives bad results, as explained in 
Appendix A, after Eq. (A10). It can be concluded that the second and third harmonics play an important role in the value of the playing frequency. Moreover, although the excitation is ignored in Eq. (25), this calculation gives a qualitative agreement with the complete computation of the oscillations.

The second method is an analytical approximation of Eq. (25), which is satisfactory for one harmonic, but for two and three harmonics, it is satisfactory only for long length $\ell\left(\ell>>x_{1}\right)$, i.e., when the resonance frequencies are low. It gives the following approximation:

$$
z \simeq x_{1} \frac{\pi^{4} \beta^{4}}{45} \frac{1+16 \cos ^{2}(\pi \beta)+9\left[3-4 \sin ^{2}(\pi \beta)\right]^{2}}{1+\cos ^{2}(\pi \beta)+\left[3-4 \sin ^{2}(\pi \beta)\right]^{2} / 9}
$$

The three terms of the numerator and the denominator correspond to the first three terms of Eq. (25).

Finally, using Eq. (A10), the inharmonicity between the first two resonance frequencies can be calculated from the ratio of the two frequencies:

$$
\frac{f_{2}}{2 f_{1}}=\frac{\ell+x_{1}-z_{1}}{\ell+x_{1}-z_{2}}=\frac{45-\pi^{4} \beta^{5}}{45-16 \pi^{4} \beta^{5}} .
$$

This gives $8 \%$ (more than a semi-tone) for the shortest length considered $(0.35 \mathrm{~m})$, and $1 \%$ for the longest length $(0.67 \mathrm{~m})$. As a consequence, the choice of the mouthpiece volume reduces the inharmonicity, but inharmonicity remains important.

\section{Analytical study of the trans- fer functions for the harmon- ics of the playing frequency}

In order to investigate the spectrum of the acoustic quantities, we need to calculate their values at the harmonics of the playing frequency. The antiformants of the input pressure and flow rate correspond to the frequencies of the minima and maxima of the input impedance sampled at the harmonics of the playing frequency.

\subsection{Input impedance extrema for the harmonics of the playing fre- quency}

When the length correction for the playing frequency is ignored (or independent of the length $\ell$ ), it was noticed in [23] that, for the harmonics of the playing frequency, the frequencies of some extrema of the sampled input impedance are independent of the cone length, i.e., of the note. Indeed, for the harmonics of the playing frequency, $f=n c / 2\left(\ell+x_{1}-z\right)$, i.e., $k \ell=n \pi-k\left(x_{1}-z\right)$, the following equation can be written as:

$$
\cot (k \ell)=-\cot \left(k\left(x_{1}-z\right)\right) .
$$

If $z$ is independent of the length $\ell$, the latter disappears in the expressions of the zeros of the transfer functions. The values of the impedance for the harmonics of the playing frequency are located on the following curve:

$$
Z=\frac{\rho c}{S_{1}} \frac{j \sin \left(k\left(x_{1}-z\right)\right)}{-\cos \left(k\left(x_{1}-z\right)\right)+\sin \left(k\left(x_{1}-z\right)\right) H\left(k x_{1}\right)} .
$$

where $H\left(k x_{1}\right)=\left[1 /\left(k x_{1}\right)-k x_{1} / 3\right]$. Therefore the extrema of this expression do not depend on $\ell$ and are common to all notes. They correspond to the zeros of the following equations, derived from Eqs. (21 and 22) with Eq. (28):

$$
\begin{aligned}
\tan \left(k\left(x_{1}-z\right)\right) & =0 \\
\cot \left(k\left(x_{1}-z\right)\right) & =1 / k x_{1}-k x_{1} / 3 .
\end{aligned}
$$

The first equation gives the frequencies of the impedance minima, while the second gives those of the impedance maxima.

What happens if $z$ is slowly varying with the length $\ell$ ? The corresponding extrema vary little with $\ell$. Figure 5 shows the input impedance modulus for the harmonics of the playing frequency. The results for 25 values of the length are superimposed. A dotted line shows an example of input impedance for a given note. The length correction $-z$, as numerically calculated in Section 4, slightly varies with the length $\ell$, so do the values of the frequencies of the extrema. They are included in a small range. This enlarges the formants and anti-formants of the impedance curve sampled at the harmonics of the playing frequencies.

In the next subsections the values of the zeros of the transfer functions, i.e., the solutions of Eqs. (30) and (31), are investigated. The zeros of Eq. (30) give the anti-formants of the input pressure, while the zeros of Eq. (31) give the anti-formants of the flow rate.

In order to obtain more general results, we extend the model of the resonator. The mouthpiece is assumed to remain lumped and lossless, with a volume equal to $\eta S_{1} x_{1} / 3$ (for $\eta=1$, it is that of the missing cone), but an acoustic mass $M_{m}=\sigma \varrho x_{1} / S_{1}$ is added (for $\sigma=1$, this is that of a cylinder of length $x_{1}$ and cross section area $S_{1}$ ). Adding an acoustic mass does not make the calculation of the resonator more complicated, while the complete computation algorithm for the oscillations should be more complicated. It is the reason why the model extension is limited to this section. Eqs. (30) and (31) are replaced by the following:

$$
\begin{aligned}
-1 /\left(\sigma k x_{1}\right) & =-\cot \left(k\left(x_{1}-z\right)\right)+1 /\left(k x_{1}\right) \\
k x_{1} \eta / 3 & =-\cot \left(k\left(x_{1}-z\right)\right)+1 /\left(k x_{1}\right) .
\end{aligned}
$$




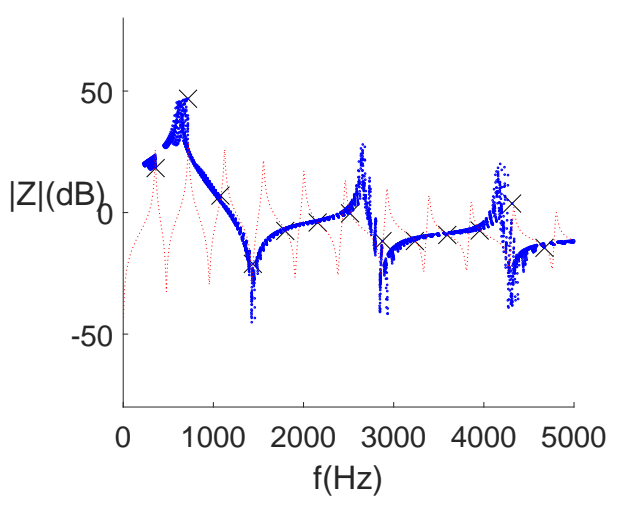

Figure 5: Values of the input impedance for the harmonics of 25 fundamental frequencies included in the first register of a soprano saxophone (thick points, blue online), corresponding to 25 values of the truncated cone length $\ell$. The impedance is calculated from Eq. (8), and plotted in dB: $20 \log \left(\left|Z S_{1} / \rho c\right|\right)$. The frequency is in Hz. The calculation of the playing fundamental frequencies uses the results presented in Fig. 4 for $\gamma=0.4 ; \zeta=0.65$. In order to exhibit an example, the results for one length is indicated by a cross ' $\mathrm{X}$ ' for $\ell=0.352 \mathrm{~m}$, and the complete impedance curve for this length is drawn by a thin line (red online).

These equations correspond to the equality of the admittances (divided by the factor $j \rho c / S_{1}$ ), when projected on the two sides of the junction. The output of the mouthpiece is on the left-hand side, while the input of the truncated cone is on the right-hand side. For Eq. (32), the input impedance of the mouthpiece vanishes, i.e., it goes though a minimum, while for Eq. (33), it is infinite, i.e., it goes through a maximum. Using Eq. (28), the parameter $\ell$ has been substituted by the parameter $z$. In the following subsections, approximated solutions of Eqs. (32) and (33) are sought with respect to $z$ and $\sigma$ or $\eta$ as:

$$
\left.k x_{1}=n \pi(1+\varepsilon)\right),
$$

where $\varepsilon$ is a small unknown. Therefore

$$
\tan \left(k\left(x_{1}-z\right)\right) \simeq n \pi\left(\varepsilon-z / x_{1}\right)
$$

after expanding the tangent function to the first order in $\varepsilon$ and $z / x_{1}$.

\subsection{Frequencies of the input flow rate anti-formants vs the playing fre- quencies}

The frequencies of the flow rate anti-formants (which correspond to the maxima of the sampled impedance) are first investigated by using Eqs. (33) and (35). At the first order in $\varepsilon$ and $z / x_{1}$, straightforward algebra

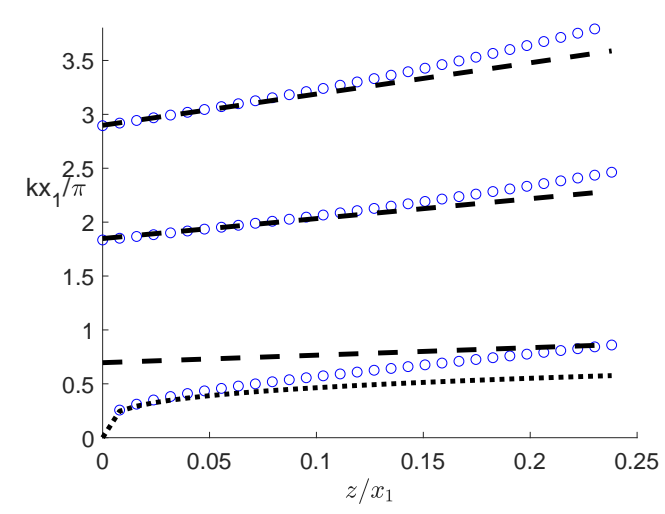

Figure 6: Frequency of the impedance maxima for the harmonics of the playing frequency with respect to the length $z . \eta=1$. Circles: numerical results of Eq. (33) (blue online); dashed lines: Eqs. (37), for $n=1,2,3$; dotted line: Eq. (38).

leads to the following result:

$$
\varepsilon=-\frac{1}{\alpha_{n}}+\frac{z}{x_{1}}\left[1-\frac{1}{\alpha_{n}}\right], \text { with } \alpha_{n}=\frac{\eta}{3} n^{2} \pi^{2} .
$$

Thus

$$
k x_{1}=n \pi\left[1-\frac{1}{\alpha_{n}}\right]\left[1+\frac{z}{x_{1}}\right]
$$

Figure 6 shows the comparison between Eq. (37) and the exact solutions of Eq. (33). The agreement of Eq. (37) with the exact result is satisfactory, except for $n=1$. For this value it is found that when $z / x_{1}$ is small, the quantity $\varepsilon$ is not small (equal to $-1 / 3$ ). For $n=1$ and small $z / x_{1}$ the formula (37) needs to be replaced by the solution of Eq. (A10) of Appendix A, as follows:

$$
k x_{1}=\left(45 z / x_{1}\right)^{1 / 4}
$$

if $\eta=1$. Fig. 6 shows the case $\eta=1$. Similar behaviour is found when the mouthpiece volume is different $(\eta \neq 1)$. Eq. (38) shows that for small $z$, there is a great variation of the frequency of the first formant. The variation of the other solutions with $z$ (for $n=2,3$ ) in Eq. (37) is significant, but narrower. As an example, for the case in study and $n=2,20 \%$ is a typical variation. This is related to the width of formants.

\subsection{Frequencies of the input pressure anti-formants vs the playing fre- quencies}

The frequencies of the pressure anti-formants (which correspond to the minima of the sampled impedance) are obtained by using Eqs. (33) and (35). The result is

$$
k x_{1}=n \pi(1+\sigma)\left(1+z / x_{1}\right)
$$




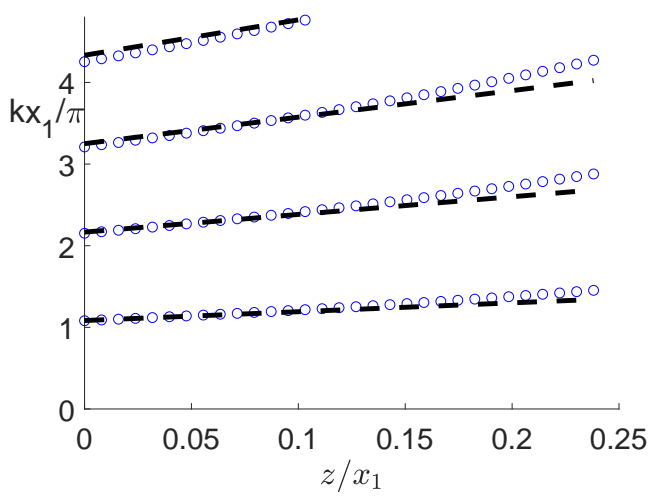

Figure 7: Frequency of the impedance minima for the harmonics of the playing frequency with respect to the length $z$. Circles: numerical results (blue online); dashed lines: Eq. (39) for $\sigma=1 / 12$.

These frequencies are also slightly higher than the values $n \pi$, which would be the values for the ideal Helmholtz motion. Moreover they vary significantly with $z$, i.e., with the playing frequency of the note played. The order of magnitude of the variation is the same as that for the flow rate. Fig. 7 compares this formula with the exact solutions of Eq. (30). The agreement is sufficient for an estimation of the influence of the pair of parameters $\left(z / x_{1}, \sigma\right)$. The value of the mouthpiece parameters have been chosen as follows: the mouthpiece is assumed to be cylindrical, with a cross section area $S_{m}=2 S_{1}$, and a volume $S_{m} \ell_{m}$ is equal to that of the missing cone length $\left(\ell_{m}\right.$ is the mouthpiece length)

$$
\sigma=\frac{S_{1}}{S_{m}} \frac{\ell_{m}}{x_{1}}=\frac{1}{3}\left(\frac{S_{1}}{S_{m}}\right)^{2}=\frac{1}{12} .
$$

For a cylindrical saxophone, the common minimum when $x_{1}$ is constant and $\ell$ varies, is given by $k x_{1}=n \pi$, i.e., $\sin \left(k x_{1}\right)=0$. Because $z=0$ for a cylindrical saxophone, this is in accordance with Eq. (39), if the acoustic mass of the small part of the cylinder is ignored.

As a conclusion, the frequencies of the antiformants of both the input pressure and the input flow rate are increasing functions of the length $z$. Furtherore the frequencies of the pressure anti-formants depend in a non negligible way on the acoustic mass of the mouthpiece.

\section{Numerical results for the spectra}

\subsection{Internal and external spectra for a given length.}

After the study of the transfer functions, we use the numerical solving of the full RTC model, including the
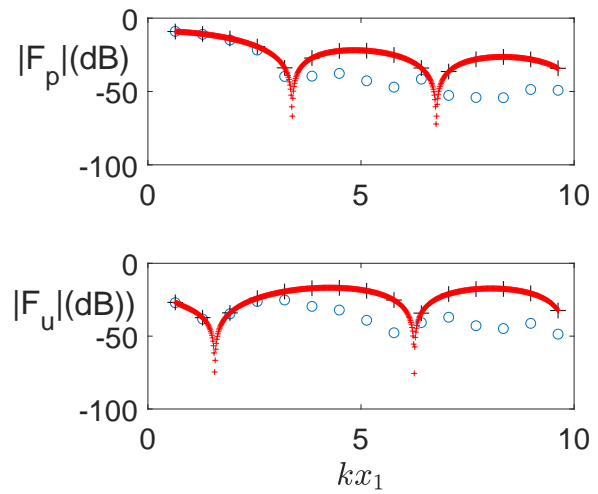

Figure 8: (top) Comparison between the input pressure $P$ (ooo, blue online) for the harmonics of the playing frequency $(275 \mathrm{~Hz})$ and the transfer function $F_{p}(+++$ black online $\left.)\right) . x_{1}=0.126 \mathrm{~m}, \ell=0.4, m \gamma=$ $0.4, \zeta=0.65$. (bottom) Comparison between the input flow rate $U$ (ooo) for the harmonics of the playing frequency and the transfer function $F_{u}(+++)$ (. The small crosses (red online) represent the transfer functions for a continuous variation of the frequency. Plot in $\operatorname{logarithic~scale:~} 20 \log \left(\left|F_{p}\right|\right)$ and $20 \log \left(\left|F_{u} \rho c / S_{1}\right|\right)$.

excitation, and find the input pressure $P$, the input flow rate $U$, and the normalized output flow rate $W$ (see Eq. (19)), which is proportional to the external pressure. The RTC model [16] gives the input quantities, and the value of the outgoing pressure wave, which is denoted $P_{2}^{+}=\mathrm{P}^{+}\left(x_{2}\right)$ (see Eq. (3)). The output flow rate can be derived as follows:

$$
U_{2}=2 \frac{S_{2}}{\rho c} P_{2}^{+}, \text {therefore } W=2 P_{1}^{+} \frac{k x_{1}}{j \gamma} .
$$

The chosen model is the simplest $(\eta=1 ; \sigma=0$, see Eqs. (32, 33)). Fig. 8 (top) shows the comparison between the spectrum modulus of the transfer function $F_{p}$ (Eq. $(21)$ ) and that of the input pressure signal $P$. For a cylindrical saxophone, because $W$ is unity (see Section 3.2), the two spectra would be identical. It appears that the effect of the cone truncation and the mouthpiece are significant, except for the first harmonics. The output flow rate cannot be infinite, therefore the zeros of the transfer function $F_{p}$ are zeros of the input pressure signal. For a better comparison between $P$ and $F_{p}$, we complete the transfer function at intermediate frequencies, by using Eq. (28), i.e., by replacing $k \ell$ by $-k\left(x_{1}-z\right)$ in the expressions $(21)$. The values at the harmonics of the playing frequency are located on this curve.

The bottom of the figure allows similar observations when comparing the transfer function $F_{u}$ (Eq. (22)) and the spectrum of the input flow rate $U$.

Fig. 9 shows the normalized output flow rate $W$. For a cylindrical saxophone, it would be equal to unity (i.e., the logarithm would vanish). In order to check 


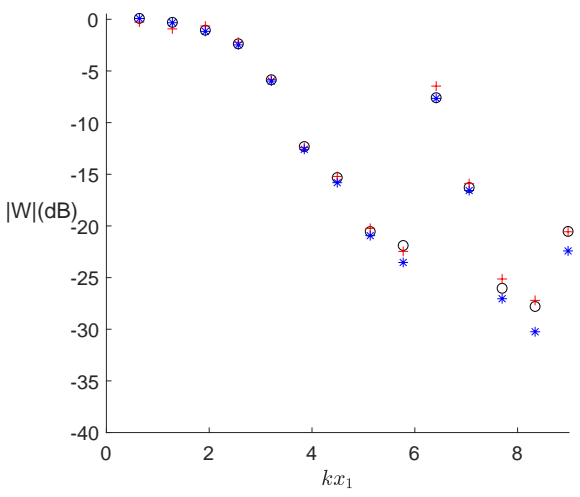

Figure 9: Normalized output flow rate $|W|$. Eq. (19) is computed in 3 ways: direct computation of the spectrum from the time-domain $(* * *$ blue online), $\left|P / F_{p}\right|$ (ooo black online), $\left|U / F_{U}\right|(+++$ red online). $\mathrm{dB}$ is $20 \log (|W|)$ (for a cylindrical saxophone, $20 \log (|W|)$ vanishes). $\quad x_{1}=0.126 \mathrm{~m}, \quad \ell=0.4, \mathrm{~m}$ $\gamma=0.4, \zeta=0.65$. the consistency of the results, the computation of $W$ was done by using the direct result of the timedomain calculation, then the computation of the ratios $\left|P / F_{p}\right|,\left|U / F_{U}\right|$. The (small) discrepancies can be due to numerical error in the determination of the playing frequency, or in the calculation of the spectra.

It appears that for higher harmonics, the flow rate is much lower than that of the Helmholtz motion. A maximum appears at $k x_{1}=6.2$. For a soprano saxophone, this corresponds to a frequency equal to 2700 Hz. Benade and Lutgen [29] found what they called "notches" in the external pressure signals, when averaged over the room of the recording. A precise comparison with our results seems to be difficult, because of the simplicity of our model. A comparison with a more complete model should be useful.

\subsection{Anti-formants in the internal spec- trum}

The transfer functions (Eq. $(21,22))$ are calculated for 32 values of the length $\ell$ and for the harmonics of the playing frequencies. The curves are superimposed in Fig. 10. Strong minima appear, therefore anti-formants can be expected in the spectra of the internal pressure and the internal flow rate. The figure 10 shows that despite the variation of the length correction $-z$ with the note played, the frequencies of the minima and maxima vary little with the note, in accordance with the results of Sect. 5. The central values of the minima depend on a unique parameter, $x_{1}$. The first ones are located at: $k x_{1}=3.4 ; 6.7 ; 10.1$ for $F_{p}$ and $1.6 ; 6.2 ; 9.9$ for $F_{u}$.

Fig. 11 is obtained with the RTC model. It confirms that anti-formants exist for the two input quantities, at the position of the minima of the transfer
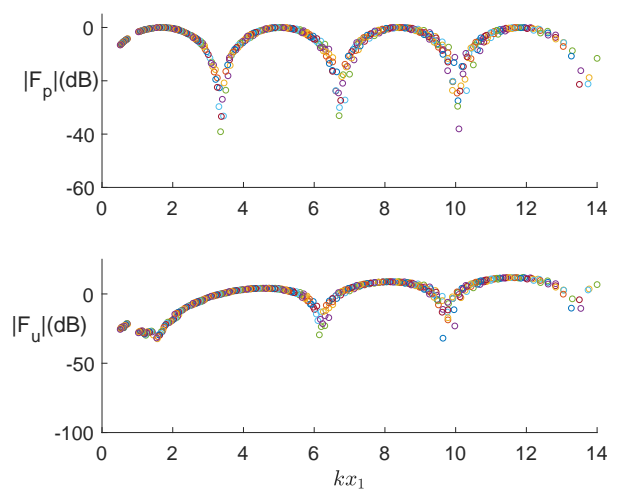

Figure 10: Transfer functions $\left|F_{p}\right|$ and $\left|F_{U}\right|$ for 32 values of the the length $\ell$. Plot in dB $20 \log \left(\left|F_{p}\right|\right)$ and $20 \log \left(\left|F_{u} \rho c / S_{1}\right|\right) \cdot x_{1}=0.126 \mathrm{~m}, \ell=0.33 \mathrm{~m}$ to $0.64 \mathrm{~m}$ $\gamma=0.4, \zeta=0.65$.
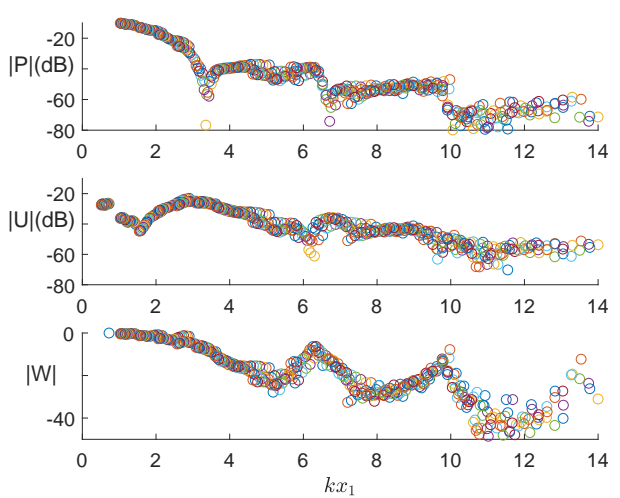

Figure 11: Input pressure $P$, input flow rate $U$, normalized output flow rate $W$ for 32 values of the length $\ell$ (in dB: $20 \log (|P|), 20 \log \left(\mid U \rho c / S_{1 \mid}\right), 20 \log (|W|)$ ). $x_{1}=0.126 \mathrm{~m}, \ell=0.33 \mathrm{~m}$ to $0.64 \mathrm{~m} \gamma=0.4, \zeta=0.65$.

functions. For a truncated cone, their width depends on the variation of the length correction with the cone length. We checked that the influence of the excitation parameters is weak.

What happens for the external spectrum, proportional to that of $W$ ? Formants seem to exist near $k x_{1}=6.2$ and 10, and maybe anti-formants near $k x_{1}=5,8$ and 11 . There is a significant difference with the anti-formants of the input quantities: we do not know the relationship with the transfer functions. It could be supposed that they depend mainly on the excitation, but this is not the case. Changing the values of the excitation parameters does not modify the general shape of the Figure 11, including the values of the extrema. Moreover the dependence on the mouthpiece volume appears to be slight. The determination of the correlation between the resonator model and the formants and anti-formants remains a topic to be investigated, but probably with a much more complete model. This will be discussed now in the light 


\section{Experimental results for the mouthpiece pressure, com- parison with the RTC model}

Decreasing chromatic scales (16 notes of the first register) were played by a saxophonist for a soprano saxophone Selmer Mark VI, an alto saxophone BuffetCrampon Senzo, and a baritone saxophone (Yanagisawa B-901). A microphone Endevco 8507-C2 is located within the mouthpiece. The Fourier analysis (FT) is done on one period, chosing a portion of each note where the pitch is rather stable.

Figure 12 shows the results for the internal pressure. The similarity of the results for the three saxophones, when scaled by the length $x_{1}$, is remarkable up to $k x_{1} \simeq 6$. This value corresponds to $2580 \mathrm{~Hz}$, $1650 \mathrm{~Hz}$, and $1080 \mathrm{~Hz}$, respectively. This confirms the essential significance of the length of the missing cone at low frequencies. Using a first order filter, we compute a smoothed value for the harmonics of different notes. These experimental results can be compared to the numerical results of Figure 11. The amplitudes of the experimental and theoretical results seem to be rather similar. However this direct comparison is not relevant, because the amplitudes depend on the excitation parameters, which were not measured for the experiment: a mezzo forte note was played with each instrument, without specific constraint for the musician. However the amplitude variation from lower to higher frequencies can be compared for the three instruments.

The frequencies of the minima (given by dotted vertical lines) are very similar for the three measured saxophones. However the frequencies given by the model are higher than the experimental ones. A reason can be the influence of the existence of taper variation, or that of the acoustic mass of the mouthpiece, because it is in series with the input impedance of the truncated cone. For simplicity, the mass is ignored in the present model, because taking the mass into account would require a very different discretized oscillation model. However, for $\sigma$ close to 0.1, Eq. (39) gives a correct order of magnitude of the necessary correction for the first frequency of minimum. Obviously, at higher frequencies, the assumption that the mouthpiece is smaller than the wavelength is questionable as well. We checked that the excitation parameters play a weak role on these frequency values.

An attempt to measure the external pressure was done, with a microphone close to the first open tonehole. However, as it is known (see e.g. [5, 29, 30, 31]), the pressure spectrum strongly depends on the location of the microphone. Above cutoff (for a discussion about the definition of the cutoff frequencies due to toneholes, see Ref. [32]), the external pressure field
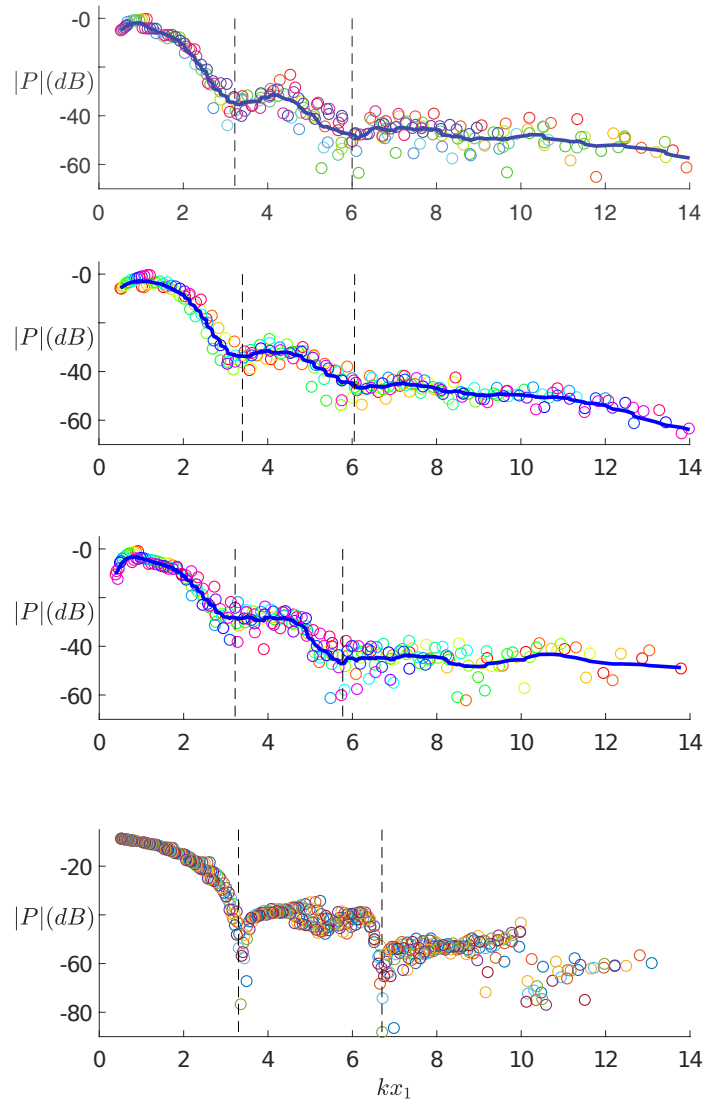

Figure 12: Mouthpiece pressure. From top to bottom: experimental results for a decreasing chromatic scale played on a soprano saxophone $\left(x_{1}=.126 \mathrm{~m}\right)$, an alto saxophone $\left(x_{1}=0.196 \mathrm{~m}\right)$ and on a baritone saxophone $\left(x_{1}=0.301 \mathrm{~m}\right)$ The abscissa is $k x_{1}$ for the different saxophones, with different $x_{1}$. Bottom: numerical results given by Fig. 11. Plot in dB: $20 \log (|P|)$. Solid, line (blue online): smoothed value of the harmonics. 
is the result of complicated interferences, and is very different from the one of a monopole. For a soprano saxophone, the cutoff can be evaluated at $1200 \mathrm{~Hz}$ $\left(k x_{1} \simeq 2.8\right):$. Moreover at this frequency the radiation by the bell is not that of a monopole $\left(k R_{2}\right.$ is close to 1.5). Notice that there are bends in baritone saxophones, therefore the interference pattern is necessarily different from that of the (straight) soprano saxophone.

These reasons are sufficient to explain why our preliminary results for the soprano and baritone saxophones are very different. In Ref. [29], the authors found that the general shapes of the external spectra can be approximated by two straight lines, crossing at $618 \mathrm{~Hz}$ for an tenor saxophone, and $837 \mathrm{~Hz}$ for an alto saxophone. The first line was increasing, while the second was decreasing. The major interest of the approach of these authors was the measurement of an average pressure in a room.

Concerning the model, it appears that the simple theoretical model is not able to give any prediction of the external spectrum. The first reason lies in the ignorance of the tonehole effects. Moreover many other phenomena intervene: boundary layer losses, radiation, reed dynamics, etc. Therefore complete study remains to be carried out, and is out of the scope of the present paper.

\section{Conclusion}

Conclusions can be drawn for the pressure spectrum in the mouthpiece:

- Anti-formants exist in the spectra of the mouthpiece pressure and input flow rate, and their frequencies are mainly related to the resonator. The values of their frequencies are related to the length of the missing cone. Formants exist as well. Their effect is less strong, but their existence can be regarded as a consequence of that of anti-formants.

- Concerning the spectra of different instruments of the saxophone family, they appear to be very similar, taken into account the scaling of the missing cone length $x_{1}$.

- The frequencies of the anti-formants are close to the natural frequencies of the missing cone length, but slightly higher. This is not in contradiction with the hypothesis that the product $k x_{1}$, i.e. the ratio of the missing cone length to the wavelength, can be regarded as a small quantity for these frequencies, but the explanation is not straightforward: it is related to the consideration of the sampling of the input impedance at the harmonics of the playing frequency. This is a major difference with a cylindrical saxophone, for which the harmonicity of the resonance frequencies is perfect, and the playing frequency is equal to that of the first impedance peak (for the simplest model).

- In other words, the difference between the inharmonicity of the resonator and the harmonicity of the spectrum in the periodic signals explain why minima exist in the input pressure and in the input flow rate.

- Furthermore inharmonicity of a conical instrument implies a variation of the negative length correction, denoted $-z$ in the present paper, when the length of the truncated cone varies. This is in particular true for the inharmonicity due to the cone truncation. A consequence is a small variation of the minimum pressure frequencies with the length of the truncated cone, i.e., with the played note, and an enlargement of the anti-formants. However, despite of this variation, existence of anti-formants is clear.

- The simplified model of [16] allows an interesting prediction of the waveshapes, and of the existence of anti-formants in the spectra of the input quantities. This is true at least up to $k x_{1} \simeq 7$., i.e., up to a ratio of the missing cone length to the wavelength equal to unity.

- Assuming a monopole radiation, the external pressure diminishes with the frequency, much more rapidly than for an ideal cylindrical saxophone (see Fig. 9) . Numerical results show that formants exist for the external spectrum and their dependence on the excitation parameters is weak. However their dependence on the geometrical parameters remains to be understood. It cannot be easily derived from that of the input quantities.

- A convincing comparison with experiment requires both a much more complete model and measurements at different microphone locations of the radiated sound.

\section{Acknowledgements}

We thank Sandra Ndayizamba very much for playing the Senzo saxophone, and the Buffet-Crampon company for lending the instrument. We thank also G. Rabau and T. Colinot for helping the preparation of the paper. This work was done in the frameworks of Labex MEC (ANR-10-LABX-0092) and of the A*MIDEX project (ANR-11-IDEX-0001-02), funded by the French National Research Agency (ANR). It was also supported by the SDNS-AIMV project financed by the ANR (project ANR-09-RPDOC-02201 ), and the LabCom LIAMFI ANR project (ANR16-LCV2-0007-01). 


\section{Appendix A: Approximate calcu- lations of the playing frequency}

The formula (24) can be rewritten by applying the residue calculus to the modal expansion of the input impedance (Eq. (8), see e.g. Ref. [19], p. 167)):

$$
Z(\omega)=\sum_{m} \frac{R e s_{m}}{\omega_{p}-\omega_{m}} .
$$

The $\omega_{m}$ 's are the poles and the Res $s_{m}$ 's are the residues. Because the input impedance is written in the form (8), which ensures that the numerator has no pole, the residues are obtained as the ratio of the numerator to the derivative of the denominator (see [19] p. 167). Because no losses are considered, the poles are real. An approximate value of $Z(\omega)$ at a given frequency can be found by truncating the series to one term only, which corresponds to the pole which is closest to this frequency. It is assumed that the frequency $\omega_{m}$ is close to $n \omega$, therefore the subscript $m$ is replaced by $n$. With this assumption, Eq. (24) becomes:

$$
\sum_{n} n\left|P_{n}\right|^{2}\left(n \omega_{p}-\omega_{n}\right) / \operatorname{Res}_{n}=0,
$$

therefore:

$$
\omega_{p}=\frac{\sum_{n} n\left|P_{n}\right|^{2} \omega_{n} / \operatorname{Res}_{n}}{\sum_{n} n^{2}\left|P_{n}\right|^{2} / \operatorname{Res}_{n}} .
$$

If all natural frequencies are harmonically related, $\omega_{n}=n \omega_{1}$, and $\omega_{p}=\omega_{1}$. Another expression can be found by defining the length corrections $z_{n}$ for the different resonance frequencies, as follows:

$$
k_{n}=\frac{\omega_{n}}{c}=\frac{n \pi}{\ell+x_{1}-z_{n}} \simeq \frac{n \pi \beta}{x_{1}}\left(1+z_{n} \frac{\beta}{x_{1}}\right) .
$$

The latter expression is valid at the first order of $z_{n} /\left(\ell+x_{1}\right)$. Using this expression, and a similar expression for $k_{p}$ derived from Eq. (23), Eq. (A2) becomes:

$$
z=\frac{\sum_{n} z_{n} n^{2}\left|P_{n}\right|^{2} / \operatorname{Res}_{n}}{\sum_{n} n^{2}\left|P_{n}\right|^{2} / \operatorname{Res}_{n}} .
$$

If the pressure spectrum is assumed to be that of the Helmholtz motion (Eq. (13)), Eq. (25) is obtained. Two calculations of the values of $z_{n}$ and $R e s_{n}$ are used: i) an exact calculation of the resonance frequencies, which are zeros of the the input impedance (Eq. (11)), and the corresponding residues; ii) an analytical approximation of these quantities.

It is possible to slightly enlarge the hypothesis for Eq. (25). Now the volume of the mouthpiece is not necessarily equal to that of the missing cone. We denote it $V=\eta x_{1} S_{1} / 3$. For the exact volume of the missing cone, $\eta=1$. In the denominator of Eq. (8), the factor $1 / 3$ is replaced by $\eta / 3$, thus the resonances are given by:

$$
\cot (k \ell)+1 /\left(k x_{1}\right)-\eta k x_{1} / 3=0,
$$

The poles are numerically computed as solutions of ${ }_{921}$ Eq. (A6). From Eq. (8), the residues are found to be:

\section{2}




$$
\operatorname{Res}_{n}^{-1}=-\frac{S_{1}}{j \omega \rho} \frac{\ell+x_{1}+k_{n}^{2} x_{1}^{2}\left(\ell\left(1-\frac{2 \eta}{3}+\frac{\eta x_{1}}{3}-\frac{2 \ell}{3} k_{n}^{2} x_{1}^{2}\right)+\eta^{2} \frac{\ell}{9} k_{n}^{4} x_{1}^{4}\right.}{k_{n}^{2} x_{1}^{2}} .
$$


where $k_{n}=\omega_{n} / c$ are numerically computed as solutions of Eq. (A6). Using Eq. (A4), the length corrections of the resonance frequencies $z_{n}$ are deduced. Then Eq. (25) is directly calculated (remember that Eq. (25) is an approximation, because the real spectrum of the input pressure is replaced by that of the Helmholtz motion). Figure 4 shows that for $\eta=1$ Eq. (25) gives lower and upper bounds for the exact values, when two and three terms of the series are taken into account. When $\eta$ is slightly different of unity, the length correction is significantly modified, but Eq. (25) remains satisfactory.

The second kind of calculation needs a further step. A first simplification is to approximate the resonance frequencies by those of the Helmholtz motion $\left(k_{n}=n \pi \beta\right)$. This is a good approximation, entailing a small error (of the second order in $z / x_{1}$ ). The second simplification is based on the approximated calculation of the length corrections $z_{n}$, by using a series expansion, as follows. From the definition (A4),

$$
\cot \left(k_{n} \ell\right)=-\cot \left(k_{n}\left(x_{1}-z_{n}\right)\right) .
$$

Therefore Eq. (A6) can be rewritten as:

$$
\cot \left(k_{n}\left(x_{1}-z_{n}\right)\right)=+\frac{1}{k x_{1}}-\frac{\eta k x_{1}}{3} .
$$

If the argument of the cotangent function is small, the following expansion can be used: $\cot (x) \simeq 1 / x-$ $x / 3-x^{3} / 45$. At this order of the cotangent function and at the first order in $z_{n} / x_{1}$ (see Eq. (A4)), this leads to the following result:

$$
z_{n} / x_{1}=\frac{k_{n}^{2} x_{1}^{2}}{3}\left[1-\eta+\frac{k_{n}^{2} x_{1}^{2}}{3}\left(\frac{1}{5}+\eta-1\right)\right] .
$$

The order of the expansion limits the value of $n k_{1} x_{1} \simeq n \pi \beta$ to approximately unity. $\beta$ being smaller than unity, the following calculation is limited to $n=3$, and this implies the truncation of the series in Eq. (A2). For the case $\eta=1$, the final result is found to be:

$$
z \simeq x_{1} \frac{\pi^{4} \beta^{4}}{45} \frac{\sum_{n=1}^{3} n^{2} \sin ^{2}(n \pi \beta)}{\sum_{n=1}^{3} n^{-2} \sin ^{2}(n \pi \beta)} .
$$

We remind that the length correction is $-z$. This can be rewritten as Eq. (26). Equations (A3) and (A5) can be used for other causes of inharmonicity. For that purpose, it could be interesting to analyse in details all causes of inharmonicity, as did Debut [33] for a clarinet. As an example, the inharmonicity due to open toneholes is negative (with a positive length correction), while that due to the cone truncation is positive. Such an effect can be large for fork fingerings [34], and entails significant effect on the playing frequency.

\section{References}

[1] R.A. Smith and D.M.A. Mercer, Possible causes of woodwind tone colour, J. of Sound Vib. 32, 347-358 (1974)

[2] W. Voigt. Research on the formant formation in sounds of dulcians and bassoons, Kölner Beitäge zur Musikforschung, Band 5 (in German), Gustav Bosse (1975)

[3] A.H. Benade, Wind instruments and music acoustics. In Sound generation in winds strings computers, Royal Swedish Academy of Music No. 29, 15-99 (1980).

[4] F. Fransson, The source spectrum of double-reed wood-wind instruments, Part 1. The bassoon, Quarterly Progress and Status Report, Dept. for Speech, Music and Hearing, 8, 35-37 (1967).

[5] D.H. Keefe, Woodwind tone hole acoustics and the spectrum transformation function, $\mathrm{PhD}$, Case Western Reserve University (1981)

[6] J. Saneyoshi, Theory on Anti-resonance Frequencies of Input Impedance of Conical Horns, --- Oscillation Frequencies of Wind Instruments with a Conical Pipe (in Japanese), Proc. of the Acoustical Society of Japan, Autumn Meeting, 65-66 (1977)

[7] A. Ya. Gokhshtein, Pressure jumps in the reflection of a wave from the end of a tube and their effect on the pitch of sound, Sov. Phys. Doklady 25, 462-464 (1980).

[8] J. P. Dalmont, J. Kergomard: Elementary model and cxperiments for the Helmholtz motion in conical woodwinds. Proceedings of the International Symposium of Musical Acoustics, Dourdan, 114-120 (1995)

[9] J.-P. Dalmont, J. Gilbert, and J. Kergomard, Reed Instruments, from small to large amplitude periodic oscillations and the Helmholtz motion analogy, Acustica united with Acta Acustica 86, 671-684 (2000).

[10] S. Ollivier, J.-P. Dalmont, and J. Kergomard, Idealized Models of Reed Woodwinds. Part I: Analogy with the Bowed String, Acta Acustica united with Acustica, 90, 1192-1203 (2004).

[11] S. Carral and V. Chatziioannou, Influence of the cone parameters on the sound of conical woodwind instruments, Proceedings of the ISMA, 201206, Le Mans, France (2014).

[12] J.-B. Doc, C. Vergez, P. Guillemain, J. Kergomard, Sound production on a "coaxial saxophone", J. Acoust. Soc. Am., 140, 3917-3924 (2016). 
[13] E.J. Irons, On the fingering of conical instruments. The London, Edinburgh, and Dublin Philosophical Magazine and Journal of Science, 11, 535-539, Iss. 70 (1931).

[14] A.H. Benade, On woodwind instruments bores. J. Acoust. Soc. Am. 31, 137-146 (1959)

[15] C.J. Nederveen, Acoustical aspects of woodwind instruments. Northern Illinois (1983).

[16] J. Kergomard, P. Guillemain, F. Silva and S. Karkar, Idealized digital models for conical reed instruments, with focus on the internal pressure waveform. J. Acoust. Soc. Am. 139, 927-937, (2016).

[17] A. H. Benade and W. Bruce Richards, Oboe normal mode adjustment via reed and staple proportioning, J. Acoust. Soc. Am. 73, 1794-1803 (1983).

[18] J.P. Dalmont, B. Gazengel, J. Gilbert, J. Kergomard, Some aspects of tuning and clean intonation in reed instruments. Applied Acoustics 46, 19-60 (1995).

[19] A. Chaigne and J. Kergomard, Acoustics of musical instruments, Springer (2016).

[20] P. Guillemain, C. Vergez, D. Ferrand, A. Farcy, An Instrumented Saxophone Mouthpiece and its Use to Understand How an Experienced Musician Plays. Acta Acustica united with Acustica 96, 622-634 (2010).

[21] T. Wilson and G. Beavers, Operating modes of the clarinet, J. Acoust. Soc. Am. 56, 653-658 (1974).

[22] J.P. Dalmont, B. Gazengel, J. Kergomard, Scaling of reed instruments: The case of the saxophone family. J. Acoust. Soc. Am. 119, 3259 (2006).

[23] J.Kergomard, Ph. Guillemain, S. Karkar, J.P. Dalmont, B. Gazengel, What we understand today on formants in saxophone sounds? $44^{\mathrm{O}}$ Congréso Español de Acústica, 1209-1216, Valladolid (2013).

[24] A.H. Benade, Fundamental of musical acoustics, Oxford University Press (1976).

[25] W. Worman: Self-sustained nonlinear oscillations of medium amplitude in clarinet likesystems. Ph.D. thesis, Case Western Reserve University Cleveland (1971).

[26] W. Coyle, P. Guillemain, J. Kergomard and J.P. Dalmont. Predicting playing frequencies for clarinets: A comparison between numerical simulations and simplified analytical formulas, J. Acoust. Soc. Am., 138, 2770-2781 (2015).
[27] B. Ricaud, P. Guillemain, J. Kergomard, F. ${ }_{1070}$ Silva, C. Vergez, Behavior of reed woodwind 1071 instruments around the oscillation threshold, 1072 Acta Acustica united with Acustica, 95,733-743 1073 (2009).

[28] X. Boutillon, Analytical investigation of the flat- ${ }_{1075}$ tening effect - the reactive power balance rule, J. $\quad 1076$ Acoust. Soc. Am. 90, 754-763 (1991).

[29] A.H. Benade and S. J. Lutgen, The saxophone spectrum, J. Acoust. Soc. Am. 83, 1900-1907 (1988).

[30] J. Kergomard, Internal and external field of wind 1081 instruments (in French), Phd, University Paris 61082 (1981).

[31] A.H. Benade, From Instrument to Ear in a 1084 Room: Direct or via Recording. J. Audio Eng. Soc. 33, 218-233 (1985).

[32] E. Moers and J. Kergomard, "On the Cutoff Frequency of Clarinet-Like Instruments. Geometrical versus Acoustical Regularity", Acta Acustica united with Acustica, 97, 984 - 996 (2011).

[33] V. Debut, J. Kergomard, and F. Laloë, "Analysis and optimisation of the tuning of the twelfths for a clarinet resonator," Appl. Acoust. 66, 365-409 (2005).

[34] C. J. Nederveen and J.-P. Dalmont, "Mode locking effects on the playing frequency for fork fingerings on the clarinet", J. Acoust. Soc. Am. 131, 689-697 (2012). 072 